\title{
Homozygous deletion in MYL9 expands the molecular basis of megacystis-microcolon-intestinal hypoperistalsis syndrome
}

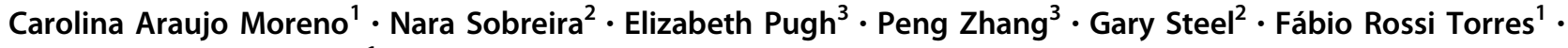 \\ Denise Pontes Cavalcanti ${ }^{1}$
}

Received: 16 June 2017 / Revised: 14 November 2017 / Accepted: 18 November 2017 / Published online: 16 February 2018

(c) European Society of Human Genetics 2018

\begin{abstract}
Megacystis-microcolon-intestinal hypoperistalsis syndrome (MMIHS) is a severe disease characterized by functional obstruction in the urinary and gastrointestinal tract. The molecular basis of this condition started to be defined recently, and the genes related to the syndrome (ACTG2-heterozygous variant in sporadic cases; and MYH11 (myosin heavy chain 11), LMODI (leiomodin 1) and MYLK (myosin light chain (MLC) kinase)-autosomal recessive inheritance), encode proteins involved in the smooth muscle contraction, supporting a myopathic basis for the disease. In the present article, we described a family with two affected siblings with MMIHS born to consanguineous parents and the molecular investigation performed to define the genetic etiology. Previous whole exome sequencing of the affected child and parents did not identify a candidate gene for the disease in this family, but now we present a reanalysis of the data that led to the identification of a homozygous deletion encompassing the last exon of MYL9 (myosin regulatory light chain 9) in the affected individual. MYL9 gene encodes a regulatory myosin MLC and the phosphorylation of this protein is a crucial step in the contraction process of smooth muscle cell. Despite the absence of human or animal phenotype related to MYL9, a cause-effect relationship between MYL9 and the MMIHS seems biologically plausible. The present study reveals a strong candidate gene for autosomal recessive forms of MMIHS, expanding the molecular basis of this disease and reinforces the myopathic basis of this condition.
\end{abstract}

\section{Introduction}

Megacystis-microcolon-intestinal hypoperistalsis syndrome (MMIHS) was initially described by Berdon et al. [1] and is characterized by a functional obstruction in the urinary and gastrointestinal tract [2]. Although the three main features of this syndrome (megacystis, microcolon, and intestinal

Electronic supplementary material The online version of this article (https://doi.org/10.1038/s41431-017-0055-5) contains supplementary material, which is available to authorized users.

$\triangle$ Denise Pontes Cavalcanti

denisepcavalcanti@gmail.com

1 Department of Medical Genetics, Faculty of Medical Sciences, State University of Campinas, Campinas, Brazil

2 McKusick-Nathans Institute of Genetic Medicine, Department of Pediatrics, Johns Hopkins University School of Medicine, Baltimore, MD, USA

3 Center for Inherited Disease Research (CIDR), JHUSOM, Baltimore, MD, USA hypoperistalsis) are often described in affected individuals, there are accumulated evidence that while gastrointestinal dysfunction seems to be usually present, microcolon does not appear to be an obligatory finding [3]. The etiology of this condition is heterogeneous and has started to be defined in recent years [4-8]. Most of the cases are sporadic and caused by de novo heterozygous variants in ACTG2 (gamma-2 actin) $[4,5]$. However, for many years, the occurrence of MMIHS in the offspring of consanguineous parents and/or the recurrence in siblings of healthy parents lent support to the hypothesis of autosomal recessive (AR) inheritance [9]. Indeed, more recently homozygous variants in MYH11 (myosin heavy chain (MHC) 11), as well as in LMODI (leiomodin 1) and MYLK (MLC kinase) were found in some patients with this condition proving the AR inheritance pattern [6-8]. These four genes related to MMIHS encode proteins involved in smooth muscle contraction, supporting a myopathic basis for the disease.

Here, we present a consanguineous family with two affected children and whose molecular investigation has led to the identification of a homozygous deletion in a novel 
A

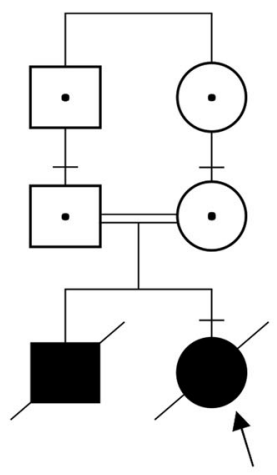

B

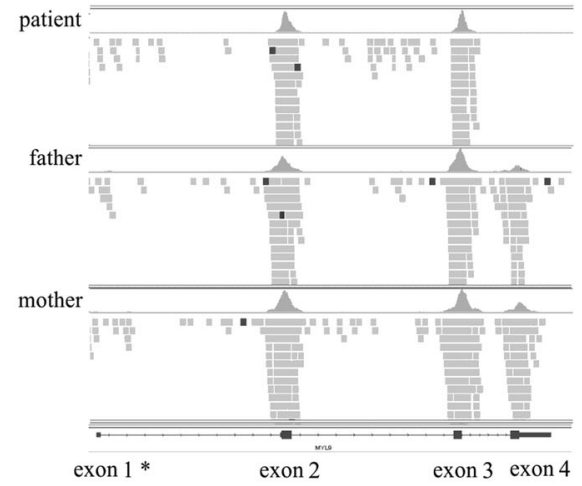

C

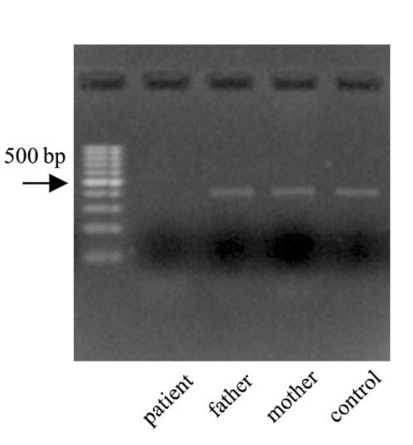

D

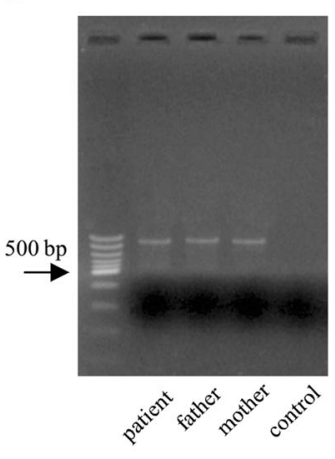

$\mathbf{E}$

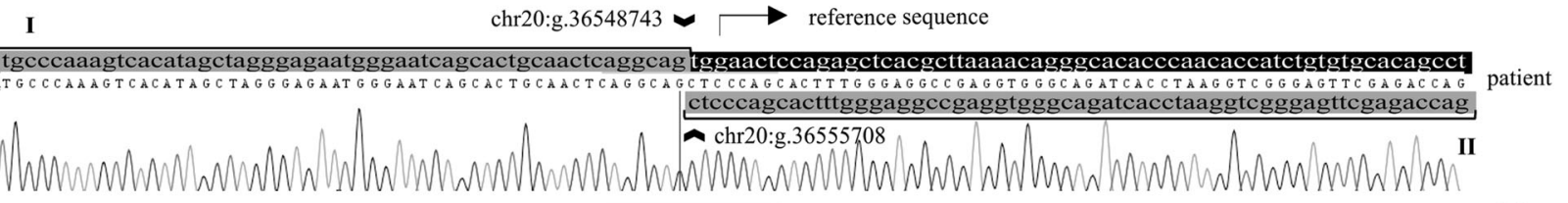

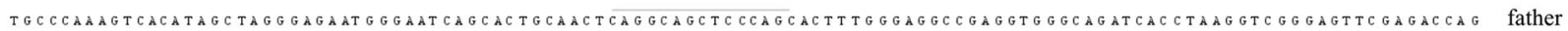

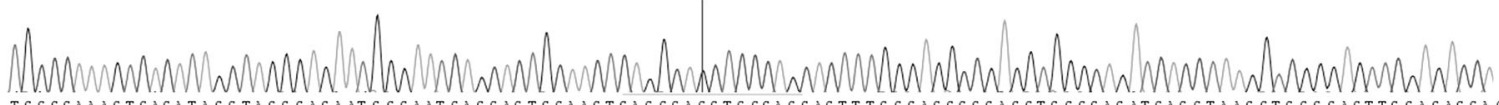

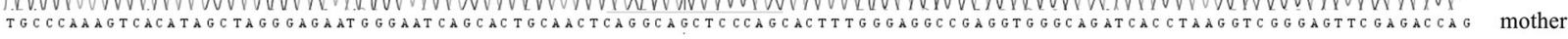

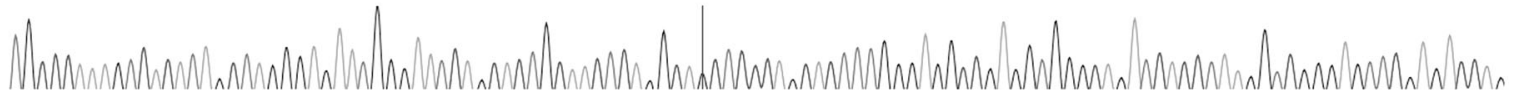

Fig. 1 Pedigree and molecular investigation of the homozygous deletion in MYL9 gene. a Pedigree shows two affected siblings with MMIHS born from consanguineous parents $(F=16)$, and identifies the individuals molecularly tested. b Integrative Genomics Viewer (IGV) view of WES trio (patient and her parents) showing no reads in exon 4 of the MYL9 gene in the patient and a reduced number of reads in both parents suggesting a homozygous and heterozygous deletions, respectively. ${ }^{*}$ There are no reads in exon 1 of the MYL9 gene, because it was not included in the capture. c Electrophoresis showing the PCR amplicon of exon 4 (407 bp) in parents and control subject. No amplification was observed in the patient. d Electrophoresis showing PCR amplicon (954 bp) using primers $2 \mathrm{~F}-5 \mathrm{R}$ that were designed to

gene, MYL9 (myosin regulatory light chain 9), which is also associated with the smooth muscle contraction.

\section{Subjects and methods}

The present study was approved by both National Committee for Ethics in Research-CONEP (protocol number 25031514.8.0000.5404) and Johns Hopkins Medicine Institutional Review Board. Written informed consent was provided by the parents on behalf of themselves and their children.

We have performed the molecular investigation of a consanguineous family with two siblings with MMIHS, who were negative for relevant alterations on whole exome sequencing (WES) in the initial analysis [3]. Due to the absence of suitable DNA of the first child (a male infant), the molecular tests were performed only in his sister (the amplify the boundaries of deletion breakpoints in the patient and her parents. No amplification of the wild-type fragment (7918 bp) was observed in the control individual because the protocol used for the PCR does not amplify fragments $>5 \mathrm{~kb}$. e Sanger sequencing of amplicon shown in d identifies a deletion of $6964 \mathrm{bp}$ (chr20: g.36548744_36555707del). The chromatogram illustrates the breakpoints of the deletion: the sequence agrees with the reference until the genomic coordinate chr20:g.36548743 (flanking region—green box I). After that, the reference sequence (flanking region-black box) is replaced by amplicon sequence which align from coordinate chr20: g.36555708 to chr20:g.36556186 (flanking region-green box II)

proband) and their parents. The DNA from the brother had been obtained from formalin-fixed paraffin-embedded tissue; however, it was inappropriate for WES, as well as for the other analysis due to its degradation. The new approach used for the reanalysis of the WES data, as well as the additional molecular investigation necessary for characterizing the candidate gene, are summarized as follows.

\section{Whole exome sequencing}

Genomic DNA was extracted from the peripheral blood of the proband, mother and father. The protocol for WES is described elsewhere [3]. The data were analyzed prioritizing homozygous deletions. This analysis was performed using the WES data from 790 samples sequenced as part of the Baylor-Hopkins Center for Mendelian Genomics, including this family. We used the Depth of Coverage (DOC) as 


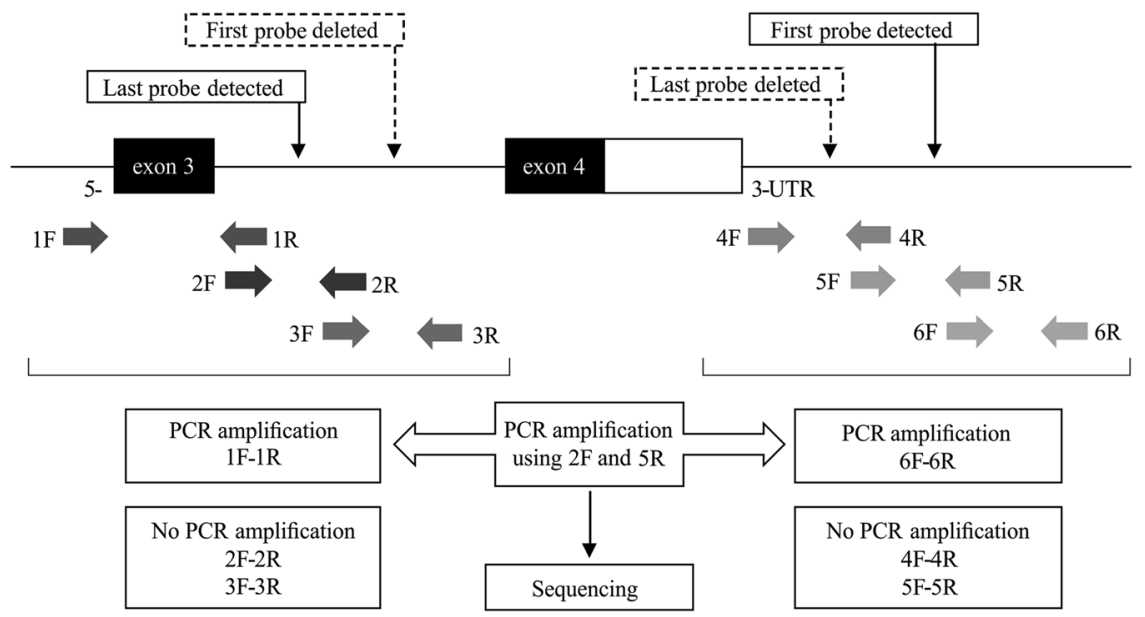

Fig. 2 Strategy used to define the breakpoints of the deletion encompassing the last exon of MYL9 gene (exon 4) 5'-region: primers named $1 \mathrm{~F}$ and $1 \mathrm{R}$ (blue arrows) were designed to anneal in a genomic region upstream from the last probe detected by CytoScan ${ }^{\circledR} \mathrm{HD}$ array. The subsequent primer pairs $(2 \mathrm{~F}$ and $2 \mathrm{R}$-red arrows; $3 \mathrm{~F}$ and $3 \mathrm{R}$-yellow arrows) were designed toward the deleted region ( $2 \mathrm{~F}$ is complementary and reverse to $1 \mathrm{R}, 3 \mathrm{~F}$ is complementary and reverse to $2 \mathrm{R}$ ); $3^{\prime}$-region: primers named as $6 \mathrm{~F}$ and $6 \mathrm{R}$ (gray arrows) were designed to anneal in a genomic region downstream from the first probe detected by CytoScan ${ }^{\circledR} \mathrm{HD}$ array. The primers pairs named $4 \mathrm{~F}$ and $4 \mathrm{R}$ (orange

calculated by GATK rescuing only the high-quality reads (minimum mapping quality score of 20). The SAS software was used to calculate the average DOC across all samples for each target region, as well as the number of regions overall and within families that had an average coverage of zero $(\mathrm{DOC}=0)$. We prioritized target regions that presented $\mathrm{DOC}=0$ that were unique to the studied family.

\section{Additional molecular investigation}

To follow up the exome reanalysis, the proband was submitted to chromosomal microarray analysis (CMA), using the CytoScan ${ }^{\circledR}$ HD Array kit (Affymetrix Inc., Santa Clara, CA, USA), according to the manufacturer's instructions. In addition, Sanger sequencing was performed in order to confirm and precisely define the boundaries of the variant identified in the candidate gene. Details regarding Sanger sequencing are described in the supplementary information (SI-1). Primers were designed based on the chromosome 20 sequence defined by GRCh38 genome reference. The variant was described using NC_000020.11 as a reference sequence. The ENST reference sequence was used to identify the transcripts of the candidate gene.

The variant information and phenotype have been submitted to the public database ClinVar (patient ID SCV000598634; https://www.ncbi.nlm.nih.gov/clinvar/). arrows) and $5 \mathrm{~F}$ and $5 \mathrm{R}$ (green arrows) were designed toward the deleted region ( $4 \mathrm{R}$ is complementary and reverse to $5 \mathrm{~F}, 5 \mathrm{R}$ is complementary and reverse to $6 \mathrm{~F}$ ). PCR amplification occurred for all primer pairs in the control individual. No PCR amplification was observed using $2 \mathrm{~F}-2 \mathrm{R}, 3 \mathrm{~F}-3 \mathrm{R}, 4 \mathrm{~F}-4 \mathrm{R}$ and $5 \mathrm{~F}-5 \mathrm{R}$ primers in the patient sample; however, PCR was successful for primers $1 \mathrm{~F}-1 \mathrm{R}$ and $6 \mathrm{~F}-6 \mathrm{R}$ (not shown). Amplicon containing the deleted region was obtained using primers $2 \mathrm{~F}$ and $5 \mathrm{R}$. This amplicon was submitted to Sanger sequencing. F forward primer, R reverse primer

\section{Results}

The studied family (Fig. 1a) was previously described in detail [3]. As with her brother, the proband described here manifested the first symptoms during the prenatal period (hydronephrosis and megacystis). Further investigation demonstrated that she had the classical triad of MMIHSmegacystis, microcolon, and intestinal hypoperistalsis, plus congenital mild mydriasis with pupils slightly reactive to the light reflex. This child was followed up to 6 months of age, when she was transferred to another country in order to attempt multi-visceral transplantation. Unfortunately, pupillary response could not be evaluated in her brother.

Reanalyzing WES data, two unique regions with DOC $=0$ were identified. Using the Integrative Genomics Viewer software, only a deletion of exon 4 in MYL9 gene (ENST00000279022.6) was considered a true variant. For this target, no reads were identified in exon 4 of the proband, while for both parents a significant reduction in the number of reads was mapped to this region (Fig. 1b). This finding is consistent with a homozygous deletion in exon 4 of MYL9 gene that was inherited from the heterozygous parents. In an attempt to validate the deletion identified by WES, we performed PCR followed by Sanger sequencing of the coding exons (2, 3, and 4) of MYL9. Amplification of exons 2 and 3 of MYL9 was observed in the patient, parents, and in a control subject. However, PCR failed to amplify exon 4 in the patient (Fig. 1c), reinforcing the hypothesis of 
homozygous deletion of this exon. To better define the breakpoints of the deletion, the patient's DNA was submitted to CMA that revealed a $7.7 \mathrm{~kb}$ deletion. This deletion spans a genomic region from normal probe profile in the beginning of intron 3 of MYL9 (g.36,548,287) to the intergenic region (g.36,556,729). Thereafter, we used the genomic coordinates from a range between the first and the last non-deleted probes that were localized in the boundaries of the deleted region to design primers with a view to determining the exact length of the deletion and its breakpoints. The sequential PCR strategy (Fig. 2) showed that PCR amplification occurred for all primer pairs in a control subject. However, PCR failed to amplify the fragments from primer pairs $2 \mathrm{~F}-2 \mathrm{R}, 3 \mathrm{~F}-3 \mathrm{R}, 4 \mathrm{~F}-4 \mathrm{R}, 5 \mathrm{~F}-5 \mathrm{R}$ and was normal for primers $1 \mathrm{~F}-1 \mathrm{R}$ and $6 \mathrm{~F}-6 \mathrm{R}$ in the patient. A 954-bp PCR product was amplified in the patient using the 2F-5R primer pair (Fig. 1d). The sequencing of this amplicon led to an accurate characterization of the deletion: chr20:g.36548744_36555707del). It starts in intron 3 and includes the whole of exon 4 and part of the intergenic region (to $4260 \mathrm{bp}$ downstream to the 3 '-untranslated region (UTR) extremity of MYL9 gene), encompassing a total of 6964 bp (Fig. 1e). The heterozygous state of this deletion in both parents was demonstrated as follows. The exon 4 was demonstrated by WES and PCR (Figs. 1b, c) in one allele, while the deletion of the other allele was confirmed by the amplification and sequencing of the fragment obtained from 2F-5R primer pair (Figs. 1d, e). Unfortunately, it was not possible to demonstrate the homozygous deletion in the affected brother of the proband. Due to DNA degradation, the PCR did not amplify the fragment encompassing the deletion.

This deletion is predicted to disrupt the formation of an intracellular protein, that is, an isoform of the regulatory MLC, which plays a crucial role in the complex system that regulates the contraction of smooth muscle (Fig. 3). Therefore, this deletion seems biologically plausible, that is, it suggests a cause-effect relationship between MYL9 and the MMIHS.

\section{Discussion}

MMIHS is a disorder characterized by severe dysmotility in both the gastrointestinal and urinary tracts, and was previously related to heterozygous variants in ACTG2 in sporadic cases [4, 5], and with homozygous variants in MYH11, LMOD1, and MYLK in children born to consanguineous parents [6-8]. However, despite advances in the molecular investigation, some cases remain without an etiological definition $[5,10]$. In the present article, we report a family with two affected siblings with MMIHS carrying a homozygous deletion of the last exon of MYL9.
The contractile apparatus of smooth muscle is constituted by different components, with actin and myosin being the major contractile proteins [11]. Myosin class II is an isoform present in smooth muscle and is constituted by two MHCs and four MLCs. These latter are composed of two regulatory chains and two essential or non-phosphorylatable chains. Each MHC binds to a pair of MLC (one regulatory MLC and one essential MLC) [11]. The phosphorylation of the regulatory MLC is a crucial step in the contraction process because it increases the ATPase activity of MHC, enhancing the interaction between actin and myosin and promoting muscle contraction [12]. The phosphorylation of the regulatory MLC is mediated by the myosin light chain kinase, a calcium/calmodulin-dependent enzyme, which is balanced by the action of the myosin light chain phosphatase that dephosphorylates the protein [11].

The regulatory MLC of smooth muscle is encoded by $M Y L 9$, also named MLC2 [13]. This gene is located on chromosome 20q11.23 (g.36,541,484-36,551,447) and encodes two transcripts (ENST00000279022.6 and ENST00000346786.2). The longer transcript has three coding exons, while the shorter has only two coding exons, giving proteins with 172 and 118 amino acids, respectively. The deletion identified in the patient with MMIHS reported here removes the last exon from both transcripts, likely disrupting the normal transcription of the gene and resulting in an aberrant or absent protein.

A heterozygous MYL9 deletion encompassing the whole exon 4 was previously identified in two individuals out of about 2500 (1000 Genomes Project; evs3645738—https:// www.ncbi.nlm.nih.gov/dbvar/variants/esv3645738/\#Varia ntDetails), giving an allele frequency of $1 / 1250$, or a predicted homozygous frequency $\left(q^{2}\right)$ of 1/1,562,500.

Until now, MYL9 gene was not associated with any human disease neither with any phenotype in animal models. Despite this, disruption of other components of the visceral contractile apparatus that are closely related to MYL9 has been demonstrated in individuals with MMIHS. The first two genes related to this syndrome, ACTG2 [4, 5] and $M Y H 11$ [6], encode the main visceral isoform of actin and the MHC present in smooth muscle, respectively. Two genes associated with MMIHS, more recently-LMOD1 and MYLK, encode a protein involved in the actin polymerization and the enzyme required for phosphorylation of the regulatory MLC coded by MYL9, respectively [7, 8].

The affected individual evaluated in the present study had the classical findings of MMIHS. She is the second affected child of first cousin parents, and a homozygous deletion identified in MYL9 is compatible with AR inheritance. MYL9 is a gene with a crucial role in smooth muscle contraction and it is closely related to genes previously associated with the MMIHS (ACTG2, MYH11, LMOD1, and MYLK-Fig. 3c). Beside this, according to different databases (GeneCard- 


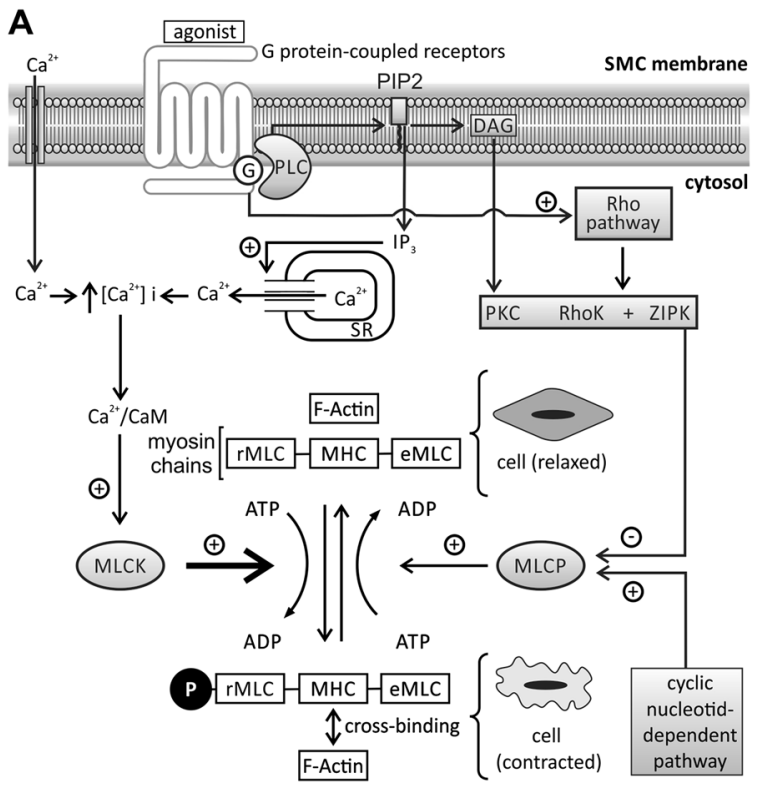

\section{B}

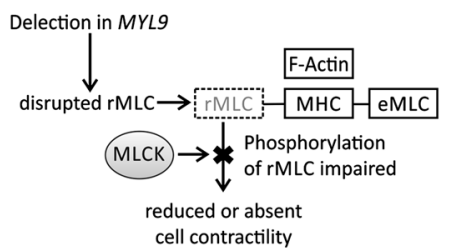

C

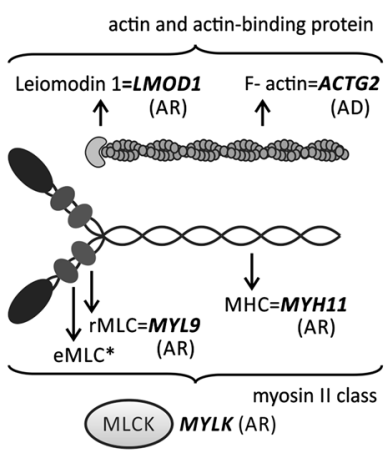

Fig. 3 Contraction of smooth muscle cell: cellular pathway and the role of MYL9 and of other genes related to MMIHS. a Diagram illustrating some steps in the cellular pathway related to smooth muscle contraction. The phosphorylation of regulatory myosin light chain (rMLC) encoded by MYL9 is crucial to promote contraction and occurs at the end of this pathway (thick arrow). The increase in intracellular calcium concentration $\left[\mathrm{Ca}^{2+}\right] \mathrm{i}$ is an essential step to initiate the process, which is triggered by several factors (autonomic nervous system, hormones, local chemical, or mechanical stimulus). The $\left[\mathrm{Ca}^{2+}\right] \mathrm{i}$ increases due to two mechanisms: the influx of calcium ions $\left(\mathrm{Ca}^{2+}\right)$ from extracellular to cytosol mediated by different channels $\left(\mathrm{Ca}^{2+}\right.$ voltage-dependent and nonselective cation channels) and by the release of this ion from sarcoplasmatic reticulum (SR) through a G-protein- (G) mediated cascade. The binding of agonists to the G-protein-coupled receptor increases the phospholipase C (PLC) activity via G-protein leading to the production of inositol 1,4,5-triphophate (IP3) and diacylglycerol (DAG) from membrane phospholipids-phosphatidylinositol 4,5-biphosphate (PIP2). Protein G also activates the Rho pathway. IP3 binds to a specific receptor (IP3R) on SR causing the release of $\mathrm{Ca}^{2+}$ to the cytosol. The activation of the ryanodine receptor (RyR) on SR also contributes to the release of $\mathrm{Ca}^{2+}$ to the cytosol and both, IPR3 and RyR, are activated by increased $\left[\mathrm{Ca}^{2+}\right] \mathrm{i}$ (not shown). The intracellular $\mathrm{Ca}^{2+}$ (four molecules) binds to calmodulin $(\mathrm{CaM})$ and this complex activates the myosin light chain kinase

http://www.genecards.org and Expression Atlas-https:// www.ebi.ac.uk/gxa/home), tissues like urinary bladder and colon, clearly affected in MMIHS, have a high mRNA and protein expression level of this gene. Based on these evidences, we suggest that MYL9 is a novel gene associated with AR MMIHS.

In the present study, despite the absence of functional studies, we hypothesize that the homozygous deletion identified in MYL9 disrupts the regulatory MLC expression in the smooth muscle cells and may compromise actin-myosin binding, thereby reducing the contraction of these smooth muscle cells, which would explain the symptoms observed in MMIHS. Additional cases of MMIHS associated with MYL9 should confirm this hypothesis.
(MLCK). Activated MLCK phosphorylates the rMLC of myosin class II (also composed by myosin heavy chain-MHC and essential myosin light chain-eMLC), allowing actin-myosin binding and generating the force necessary to contract the cell. Dephosphorylation of rMLC occurs by the action of the myosin light chain phosphatase (MLCP), inducing the relaxation. MLCP is inactivated via phosphorylation mediated by kinases (PKC, RhoK, and ZIPK-activated by DAG and Rho G pathway) and activated by the cyclic nucleotide-dependent pathway. The increase in $\left[\mathrm{Ca}^{2+}\right]$ i also stimulates the contraction by the pathway related to caldesmon, an actin-binding protein (not shown). b The presumed effect in smooth muscle contraction due to deletion in MYL9: the disruption of rMLC caused by deletion impairs the phosphorylation mediated by MLCK, leading to reduced or absent contraction. $\mathbf{c}$ The genes and the respective contractile filaments and inheritance patterns related to MMIHS-ACTG2 filamentous actin (F-actin), gamma-2 isoform, MYH11 myosin heavy chain (MHC) 11, MYLK myosin light chain kinase (MLCK), MYL9 regulatory myosin light chain (rMLC), LMOD1 leiomodin 1, an actin-binding protein. *eMLC encoded by MYL6 gene, not related to MMIHS until now, AD autosomal dominant inheritance, AR autosomal recessive inheritance. The information regarding smooth muscle contraction was based on different references $[11,28-30]$

Regarding a genotype-phenotype correlation in MMIHS, no distinct clinical features could be associated with the previously reported genes [4-8]. In order to define a possible clinical correlation in the future, a more precise characterization of the phenotype related to each gene is essential. In this way, we believe that some clinical signs such as mydriasis and vascular abnormalities should be carefully evaluated. Moreover, a more specific phenotypic characterization could highlight interesting points regarding gene expression in smooth muscle cell. Mydriasis, which was also identified in the patient reported here, was previously described in two patients with MMIHS before the first description of molecular etiology associated with this syndrome [14, 15]. Both patients had histological changes 
suggestive of smooth muscle myopathy and parental consanguinity was described in one of these families [14]. Interestingly, mydriasis was not reported in any of the patients with MMIHS carrying variants in the genes previously related to the disease [3-8, 10, 16-18]. Based on this evidence, we hypothesize that mydriasis could help to distinguish between cases related to MYL9 and other genes, since MYL9 seems to play a role in the smooth muscle contraction of the iris. It is important to note that, until now, there are few reports of individuals with MMIHS and variants in $M Y H 11, L M O D 1$, and $M Y L K$, making it difficult to evaluate the spectrum of the phenotype associated with variants in these genes. Mydriasis is also a typical sign present in another phenotype of visceral myopathy, the multisystemic smooth muscle dysfunction syndrome (MSMDS), caused by a heterozygous variant in ACTA2 [19]. ACTA2 encodes the vascular actin isoform (alpha 2) [20]. The absence of mydriasis in the ACTG2-related MMIHS and its presence in MSMDS suggests that ACTA2 is the main isoform of actin in the smooth muscle of sphincter pupillae, the muscle located in the iris whose contraction results in miosis [21]. Similarly to mydriasis, vascular involvement is a typical finding in MSMDS [19], and was not reported in any patient with MMIHS related to ACTG2 [3-8, 10, 16-18]. This difference can be explained by the predominance of the alpha-actin as the main isoform of actin expressed in vascular smooth muscle, while the gamma isoform is a minor component [22]. The vascular involvement remains as a possibility in MMIHS related to genes implicated in the AR forms. Heterozygous loss-offunction variants in MYHII and MYLK cause aortic dissection [23, 24] and $M y h 11^{-1-}$ mice [25] presented with patent ductus arteriosus. No vascular finding was described in humans with disrupted expression of LMODI nor in mice LmodI $^{-/-}$, despite the demonstration of expression of this gene in vascular tissue [7].

Although no significant vascular abnormality has been evidenced in the patient reported here, nor in her brother, it remains a possibility, as MYL9 expression has been demonstrated in vascular smooth muscle [26, 27]. Additional studies are necessary to define a possible vascular manifestation in MMIHS.

In conclusion, the findings reported here support $M Y L 9$ as a candidate gene associated with AR form of MMIHS, and expand the etiological spectrum of this syndrome. Since MYL9 encodes a protein essential for the contraction of smooth muscle cells, the present association reinforces the myopathic basis of this condition.

Acknowledgements The authors thank the family for their participation in this study, Prof. Íscia Lopes-Cendes for contributing to the CMA, Prof. Robert Pogue for reviewing the manuscript, and $\mathrm{Mr}$. Mario Moreira da Silva for helping to make the Fig. 3. The grant sponsors of this work were: National Human Genome Research
Institute-NHGRI (1U54HG006542); Conselho Nacional de Desenvolvimento Científico e Tecnológico-CNPq (590148/2011-7); Fundo de Apoio ao Ensino, à Pesquisa e Extensão-FAEPEX (0348/16).

\section{Compliance with ethical standards}

Conflict of interest The authors declare that they have no conflict of interest

\section{References}

1. Berdon WE, Baker DH, Blanc WA, Gay B, Santulli TV, Donovan C. Megacystis-microcolon-intestinal hypoperistalsis syndrome: a new cause of intestinal obstruction in the newborn. Report of radiologic findings in five newborn girls. Am J Roentgenol. 1976;126:957-64.

2. Gosemann JH, Puri P. Megacystis-microcolon intestinal hypoperistalsis syndrome: systematic review of outcome. Pediatr Surg Int. 2011;27:1041-6.

3. Moreno CA, Metze K, Lomazi EA, Bertola DR, Barbosa RH, Cosentino V, et al. Visceral myopathy: clinical and molecular survey of a cohort of seven new patients and state of the art of overlapping phenotypes. Am J Med Genet A. 2016;170:2965-74.

4. Thorson W, Diaz O, Joseph H, Ii F, Spiliopoulos M, Quintero R, et al. De novo ACTG2 mutations cause congenital distended bladder, microcolon, and intestinal hypoperistalsis. Hum Genet.. 2014;133:737-42.

5. Wangler MF, Gonzaga-Jauregui C, Gambin T, Penney S, Moss T, Chopra A, et al. Heterozygous de novo and inherited mutations in the smooth muscle actin (ACTG2) gene underlie megacystismicrocolon-intestinal hypoperistalsis syndrome. PLoS Genet.. 2014;10:e1004258.

6. Gauthier J, Ouled AB, Hamdan FF, Harrison SM, Baker LA, Couture $\mathrm{F}$, et al. A homozygous loss-of-function variant in MYH11 in a case with megacystis-microcolon-intestinal hypoperistalsis syndrome. Eur J Hum Genet. 2015;23:1266-8.

7. Halim D, Wilson MP, Oliver D, Brosens E, Verheij JB, Han Y, et al. Loss of LMOD1 impairs smooth muscle cytocontractility and causes megacystis-microcolon intestinal hypoperistalsis syndrome in humans and mice. Proc Natl Acad Sci USA. 2017;114:e273947.

8. Halim D, Brosens E, Muller F, Wangler MF, Beaudet AL, Lupski $\mathrm{JR}$, et al. Loss-of-function variants in MYLK cause recessive megacystis microcolon intestinal hypoperistalsis syndrome. Am J Hum Genet. 2017;101:123-9.

9. Mc Laughlin D, Puri P. Familial megacystis microcolon intestinal hypoperistalsis syndrome: a systematic review. Pedia Surg Int. 2013;29:947-51.

10. Halim D, Hofstra RM, Signorile L, Verdijk RM, van der Werf CS, Sribudiani Y, et al. ACTG2 variants impair actin polymerization in sporadic megacystis microcolon intestinal hypoperistalsis syndrome. Hum Mol Genet. 2016;25:571-83.

11. Sanders KM, Koh SD, Ro S, Ward SM. Regulation of gastrointestinal motility-insights from smooth muscle biology. Nat Rev Gastroenterol Hepatol. 2012;9:633-45.

12. Ikebe M, Hartshorne DJ, Elzinga M. Identification, phosphorylation, and dephosphorylation of a second site for myosin light chain kinase on the 20,000-dalton light chain of smooth muscle myosin. J Biol Chem. 1986;261:36-39.

13. Kumar CC, Mohan SR, Zavodny PJ, Narula SK, Leibowitz PJ. Characterization and differential expression of human vascular smooth muscle myosin light chain 2 isoform in nonmuscle cells. Biochemistry.. 1989;28:4027-35. 
14. Narayanan M, Murphy MS, Ainsworth JR, Arul GS. Mydriasis in association with MMIHS in a female infant: evidence for involvement of the neuronal nicotinic acetylcholine receptor. J Pediatr Surg. 2007;42:1288-90.

15. McClelland C, Walsh RD, Chikwava KR, Johnson MP, Mattei P, Liu GT. Congenital mydriasis associated with megacystis microcolon intestinal hypoperistalsis syndrome. J Neuroophthal. 2013;33:271-5.

16. Tuzovic L, Tang S, Miller RS, Rohena L, Shahmirzadi L, Gonzalez K, et al. New insights into the genetics of fetal megacystis: ACTG2 mutations, encoding $\gamma-2$ smooth muscle actin in megacystis microcolon intestinal hypoperistalsis syndrome (Berdon Syndrome). Fetal Diagn Ther. 2015;38:296-306.

17. Matera I, Rusmini M, Guo Y, Lerone M, Li J, Zhang J, et al. Variants of the ACTG2 gene correlate with degree of severity and presence of megacystis in chronic intestinal pseudo-obstruction. Eur J Hum Genet. 2016;24:1211-5.

18. Lu W, Xiao Y, Huang J, Tao Y, Yan W, Lu L, et al. Mutation in actin $\gamma$-2 responsible for megacystis microcolon intestinal hypoperistalsis syndrome in 4 Chinese patients. J Pediatr Gastroenterol Nutr. 2016;63:624-6.

19. Milewicz DM, Østergaard JR, Ala-Kokko LM, Khan N, Grange DK, Mendoza-Londono R, et al. De novo ACTA2 mutation causes a novel syndrome of multisystemic smooth muscle dysfunction. Am J Med Genet A. 2010;152A:2437-43.

20. Vanderkerckhove J, Weber K. The complete amino acid sequence of actins from bovine aorta, bovine heart, bovine fast skeletal muscle, and rabbit slow skeletal muscle. A protein-chemical analysis of muscle actin differentiation. Differentiation.. 1979;14:123-33.
21. McDougal DH, Gamlin PD. Autonomic control of the eye. Compr Physiol. 2015;5:439-73.

22. Gabbiani G, Schmid E, Winter S, Chaponnier C, de Ckhastonay C, Vandekerckhove J, et al. Vascular smooth muscle cells differ from other smooth muscle cells: predominance of vimentin filaments and a specific alpha-type actin. Proc Natl Acad Sci USA. 1981;78:298-302.

23. Zhu L, Vranckx R, Khau Van Kien P, Lalande A, Boisset N, Mathieu F, et al. Mutations in myosin heavy chain 11 cause a syndrome associating thoracic aortic aneurysm/aortic dissection and patent ductus arteriosus. Nat Genet.. 2006;38:343-9.

24. Wang L, Guo DC, Cao J, Gong L, Kamm KE, Regalado E, et al. Mutations in myosin light chain kinase cause familial aortic dissections. Am J Hum Genet. 2010;87:701-7.

25. Morano I, Chai GX, Baltas LG, Lamounier-Zepter V, Lutsch G, Kott M, et al. Smooth muscle contraction without smooth muscle myosin. Nat Cell Biol. 2000;2:371-5.

26. Licht AH, Nübel T, Feldner A, Jurisch-Yaksi N, Marcello M, Demicheva E, et al. Junb regulates art erial contraction capacity, cellular contractility, and motility via its target Myl9 in mice. J Clin Invest. 2010;120:2307-18.

27. Chen L, Fan Y, Wan J. Screening of key genes of unruptured intracranial aneurysms by using DNA microarray data analysis techniques. Genet Mol Res. 2014;13:758-67.

28. Marston SB, Redwood CS. The molecular anatomy of caldesmon. Biochem J.. 1991;279:1-16.

29. Webb RC. Smooth muscle contraction and relaxation. Adv Physiol Educ. 2003;27:201-6.

30. Fry CH, Meng E, Young JS. The physiological function of lower urinary tract smooth muscle. Auton Neurosci.. 2010;154:3-13. 